\title{
(K)EIN WELTLICH DING ZUR SPIRITUALITÄT DER EHE IN EINER ZEIT DER ORIENTIERUNGSLOSIGKEIT
}

\section{Rzecz (nie-) światowa. O duchowości małżeństwa w czasie braku orientacji}

W obecnym czasie tocza się gorace dyskusje na temat matzeństwa w spoteczeństwie, polityce i Kościele. W'śód wielu opinii i propozycji innych form życia wspólnego współczesny człowiek czuje się często zagubiony i nie wie, którego glosu powinien stuchać. Z pomoca przychodzi mu Pismo Święte. Już w Starym Testamencie malżeństwo, tzn. związek mężczyzny i niewiasty, jawi się jako dar Stwórcy i ma wartość sakralna, a w Nowym Testamencie jest znakiem miłości Chrystusa do Kościoła. Tajemnice tę wyjaśnia wielowiekowa refleksja teologiczna Kościoła.

Stowa kluczowe: duchowość matżeństwa, Kościót, Biblia.

\section{Die Ehe in der Diskussion}

Derzeit wird die traditionelle Ehe in der Gesellschaft, der Politik und der Kirche heftig diskutiert. Der Grund dafür ist, dass inzwischen viele verschiedene andere Formen von Lebensgemeinschaften in der Gesellschaft anerkannt werden. Die Politik gibt keine ethischen Vorgaben und vermittelt keine Werte in diesem Bereich, sondern geht von der ,normativen Kraft des Faktischen“ aus. Das, was es in der Gesellschaft gibt, muss auch erlaubt sein, sofern es

1 Ks. Cornelius Roth - prof. dr hab., dogmatyk, teolog duchowości, obecnie wykładowca liturgiki i duchowości na Wydziale Teologicznym w Fuldzie w Niemczech. Rektor Wyższego Seminarium Duchownego w Fuldzie; autor wielu artykułów naukowych z dziedziny duchowości. 
keinem schadet bzw. dessen Rechte verletzt (bis hin zu gleichgeschlechtlichen Lebensgemeinschaften). Dass aber durch die Gleichmachung aller bestehenden Gemeinschaften indirekt und unsichtbar viel Schaden angerichtet wird (vor allem den Kindern), wird dabei nicht berücksichtigt. Zwei Schlaglichter sollen hier zu Beginn genannt werden:

Gesellschaftspolitisch gibt es derzeit eine große Diskussion um die sog. „Homo-Ehe“. Dabei wird interessanterweise großer Wert auf die Verbindlichkeit, d.h. die lebenslange Treue zwischen den sich liebenden Partnern gelegt. Es gibt auch kirchliche Stimmen, die diese lebenslange Verbindlichkeit unter zwei Männern/Frauen honoriert sehen möchten. Die traditionelle Ehe zwischen Mann und Frau gerät so immer mehr unter Rechtfertigungsdruck. Selbst die eher als liberal anzusehende christliche Wochenzeitschrift „Christ in der Gegenwart“ stellte vor kurzem die besorgte Frage: „Was ist der Werterepublik die Ehe wert?" ${ }^{\text {2 }}$ Die ernüchternde Antwort: Scheinbar nichts. Denn Menschen (nicht nur Christen), welche die traditionelle Ehe mit Mann, Frau und Kin$\mathrm{d}(\mathrm{ern})$ gegen eine Gleichstellung anderer Lebensgemeinschaften verteidigen, wird allzu schnell die Keule der „Homophobie“ (für mich ein Kandidat für das „Unwort“ des Jahres) und Rückständigkeit um die Ohren gehauen. Abzulesen war das in Baden-Württemberg an der Initiative gegen den Bildungsplan 2015 in den Schulen des Landes, in dem nicht nur Toleranz, sondern eine bevorzugte Unterrichtsbehandlung der Lebensweisen sog. LSBTTI-Menschen gefordert wurde ${ }^{3}$. Die Möglichkeit, dass sich ein Kind/Jugendlicher auch für die „normale“ Ehe interessieren könnte, wird in dem Entwurf der Landesregierung kaum noch in Betracht gezogen. Die ,klassische“ Ehe (wie sie dann genannt wird) ist allenfalls noch eine Möglichkeit unter vielen anderen. Die Kritiker dieses Bildungsplans werden aber diffamiert und in die Ecke der Intoleranz und Homophobie gestellt.

Dabei merken nachdenkliche Leute - ganz unabhängig von ihrem Glauben oder Nichtglauben -, dass sich die Gesellschaft mit einer solchen Sicht in eine falsche Richtung bewegt und „die im Haushalt der geistigen und seelischen Ökologie minimierte Ehe mit ihrer generationenübergreifenden Verpflichtung und Verantwortung alles andere als nur Privatsache ist. Das ,weltlich Ding erweist sich, je mehr es nivelliert und missachtet wird, paradoxerweise als ein

\footnotetext{
2 J. Röser, in: CIG Nr. 4 (2014) 39-40. 43.

3 Mit der Abkürzung LSBTTI ist gemeint: Lesbisch, Schwul, Bisexuell, Transsexuell, Transgender, Intersexuell.
} 
eminent ,politisch Ding für humane Sinnstiftung und Wertebildung ${ }^{64}$. Die „klassische“ Ehe ist also nicht nur ein zu schützender Wert für die Kirche, sondern auch für den Staat und die Gesellschaft. Die zahlreichen Proteste, die es in Frankreich gegen die Einführung der Homo-Ehe gab, haben gezeigt, dass sich ganz normale Bürger, die gar nicht unbedingt christlich geprägt sind, gegen eine Nivellierung der echten Ehe zwischen Mann und Frau wehren. Der gesunde Menschenverstand führt viele zur Erkenntnis, dass es keine echte Alternative zur Einehe gibt. „Sie bleibt krisenanfällig, aber es gibt nichts Besseres und nichts Gleichwertiges. Sie bewährt sich trotz der menschlichen Schwächen und Triebstruktur immer noch als günstigste, angemessenste Institution für ein partnerschaftlich-verbindliches Liebesverhalten, für die Stabilität von Familien, zum Wohl des Nachwuchses, für gesellschaftlich-staatlich-soziale Verantwortung in Reproduktion, Erziehung, Generationenvertrag. Ehe und Familie sorgen wie keine andere Institution für das Wohl des individuellen Gemeinschaftswesens Mensch" ${ }^{\text {"5 }}$. Doch leider werden solche Stimmen immer weniger gehört.

Eine andere Infragestellung der Ehe kommt von der Kirche selbst, in Deutschland derzeit v.a. von der evangelischen Kirche (während in der katholischen Kirche nach den Umfragen zur Bischofssynode die unauflösliche sakramentale Ehe nicht als solche in Frage gestellt wird, sondern „nur“ der Umgang mit Geschiedenen, die eine neue Ehe eingegangen sind) ${ }^{6}$. Ein EKD-Papier von

4 Röser, in: CIG Nr. 4 (2014) 43.

5 Ebd., 39.

${ }^{6}$ Der Umgang der Kirche mit wiederverheiratet Geschiedenen scheint dem Papst sehr am Herzen zu liegen. Auch vor den polnischen Bischöfen hat er kürzlich bei deren Ad-Limina-Besuch darauf hingewiesen, dass die Betroffenen „nicht das Gefühl haben (sollen), dass sie von der Barmherzigkeit Gottes, der brüderlichen Liebe anderer Christen und der Sorge der Kirche um ihr Heil ausgeschlossen sind“. Gleichwohl beklagt Franziskus auch ein falsches Verständnis von Ehe als „Form von emotionaler Sonderzulage" und fordert eine gründlichere Vorbereitung auf die Ehe. Vgl. die Zitate bei http://www.domradio.de/themen/ehe-und-familie/2014-02-07/franziskus-beklagtschwindende-wertschaetzung-der-ehe (Abruf 07.03.2014). Beim Konsistorium am 22.02.2014 hat Kardinal Kasper vor den versammelten Kardinälen ein Referat mit dem Titel „Das Evangelium von der Familie“ gehalten (gekürzter Text in der FAZ vom 03.03.2014, S. 6), in dem er u.a. deutlich macht, dass er sich im Fall der Wiederverheiratet Geschiedenen Einzelfalllösungen vorstellen könnte, die sich an Aussagen und der Praxis der frühen Kirche orientieren. Denn dort gab es - etwa für Christen, die ihre Taufe in der Verfolgung verraten hatten - die Möglichkeit, nach einer Zeit der kanon- 
2013 zu Ehe und Familie, das sich selbst als „Orientierungshilfe“ bezeichnet, stiftet hier eher Verwirrung? ${ }^{7}$ Es trägt den Titel „Zwischen Autonomie und Angewiesenheit. Familie als verlässliche Gemeinschaft stärken" und kommt in seiner theologischen Analyse der Ehe zu dem Schluss:

„Die Bibel beschreibt im Alten und Neuen Testament das familiale $\mathrm{Zu}$ sammenleben in einer großen Vielfalt. Das historisch bedingte Ideal der bürgerlichen Familie kann daher biblisch nicht als einzig mögliche Lebensform begründet werden. Die evangelische Kirche würdigt die Ehe als besondere Stütze und Hilfe, die sich auf Verlässlichkeit, wechselseitige Anerkennung und Liebe gründet. Gleichzeitig ist sie gehalten, andere an Gerechtigkeit orientierte Familienkonstellationen sowie das fürsorgliche Miteinander von Familien und Partnerschaften - selbst in ihrem Scheitern - zu stärken, aufzufangen und in den kirchlichen Segen einzuschließen“ (Nr. 134).

Was das für den Umgang mit homosexuellen Paaren bedeutet, erläutert das Papier wie folgt: „Durch das biblische Zeugnis hindurch klingt als ,Grundton“ vor allem der Ruf nach einem verlässlichen, liebevollen und verantwortlichen

ischen Buße eine Art zweiter Taufe zu empfangen: nicht eine Taufe mit Wasser, sondern mit den „Tränen der Reue“. Analog dazu ist es nach Kardinal Kasper erwiesen, „dass es in einzelnen Ortskirchen das Gewohnheitsrecht gab, wonach für Christen, welche zu Lebzeiten des ersten Partners in einer zweiten Verbindung lebten, nach einer Zeit der Buße zwar keine zweite Ehe, wohl aber durch die Teilnahme an der Kommunion eine Planke des Heils zur Verfügung stand." So müsse man auch für heute fragen, ob es nicht einen Weg für Menschen geben könnte, die nach einer Ehe ihr Scheitern erkannt hätten, die sich bekehrten, die keine Verpflichtungen mehr aus der früheren Verbindung hätten, wohl aber aus der neuen - und die sich ernsthaft nach dem Sakrament der Eucharistie sehnten. Kasper argumentiert in dieser Frage auch mit der geistlichen Kommunion, die ja schon von Joseph Ratzinger als Professor und später auch als Papst für Wiederverheiratet Geschiedene ins Spiel gebracht wurde, geht aber einen Schritt weiter und fragt: „Wer die geistliche Kommunion empfängt, ist eins mit Jesus Christus; wie kann er sich dann im Widerspruch zum Gebot Christi befinden? Warum kann er dann nicht auch die sakramentale Kommunion empfangen?" Die Kirche habe in zentralen Fragen immer schon neue Türen geöffnet, ohne dabei die verbindliche dogmatische Tradition anzutasten (z.B. hinsichtlich der Ökumene auf dem II. Vatikanum), so könne auch in diesem Fall eine Änderung der Lehre in Einzelfällen (nicht generell) möglich sein. Eine großzügigere Regelung bei den Ehenichtigkeitsverfahren lehnt Kasper allerdings ab, da sie letztlich eine ,katholische Scheidung“ in getarnter Form darstellt. Letztlich geht es ihm in dieser Frage um einen „Weg jenseits von Rigorismus und Laxismus“.

7 Vgl. den Text unter http://www.ekd.de/download/20130617_familie_als_verlaessliche_gemeinschaft.pdf(Abruf 07.03.2014). 
Miteinander, nach einer Treue, die der Treue Gottes entspricht. Liest man die Bibel von dieser Grundüberzeugung her, dann sind gleichgeschlechtliche Partnerschaften, in denen sich Menschen zu einem verbindlichen und verantwortlichen Miteinander verpflichten, auch in theologischer Sicht als gleichwertig anzuerkennen. Nutzen homosexuelle Menschen heute die rechtliche Möglichkeit der eingetragenen Partnerschaft, dann erklären sie, wie heterosexuelle Menschen, bei der Eheschließung öffentlich ihren Willen, sich dauerhaft aneinander zu binden und füreinander Verantwortung zu tragen. Manches heterosexuelle Paar entscheidet sich bewusst gegen Kinder oder bleibt aus anderen Gründen kinderlos und gestaltet seine Generationenbeziehungen dennoch schöpferisch und verantwortlich. Dass homosexuelle Paare gemeinsam keine Kinder zeugen können, kann deshalb kein Grund sein, ihnen den Segen zu verweigern" (Nr. 51).

Die „Orientierungshilfe“ vermittelt mit diesen Worten den Eindruck, als ob man sich in der Kirche den Gegebenheiten in der Gesellschaft anpassen müsse ${ }^{8}$. Es ist natürlich empirisch richtig, dass sich Familie und Ehe heute anders gestalten als vor 50 oder 100 Jahren; ebenfalls ist korrekt, dass es verschiedene Formen des Zusammenlebens gibt (generationenübergreifend, gleichgeschlechtlich, Single mit wechselnden Lebensabschnittsgefährten etc.). Die Kirche sollte das wahrnehmen (auch Papst Franziskus tut dies). Aber sie muss in dieser Zeit auch Orientierung geben - nicht im Sinn eines ständigen Vorschreibens, wie es richtig zu machen sei, sondern als Wegweisung zu einem Leben, von dem sie überzeugt ist, dass es trotz vieler Krisen und des Scheiterns vieler Beziehungen dennoch für sehr viele Menschen Erfüllung und Glück bedeutet.

Die Kirche hat in diesem Zusammenhang die Aufgabe, den theologischen Sinn der christlichen Ehe herauszustellen, die Bedeutung des Sakraments zu erklären und das Bewusstsein zu schärfen, dass der Ehebund zwischen Mann und Frau eine von Gott gewollte, in der Schöpfungsordnung angelegte Institution ist, die auch für die heutige Gesellschaft ein Segen ist.

\section{Biblische Grundlagen}

8 Vgl. demgegenüber Kasper, Das Evangelium von der Familie, 6: „Deshalb kann unsere Position nicht die einer liberalen Anpassung an den Status quo sein, sondern nur eine radikale, die zurückgeht zu den Wurzeln (radices), das heißt zum Evangelium, und die von da aus nach vorne schaut.“ 
Schon im AT wird die Ehe als Schöpfungsgabe verstanden ${ }^{9}$. Im Unterschied zu anderen Kulturen, in denen die Verbindung von Mann und Frau sakralisiert wurde (als Akt kosmischer Versöhnung), hat in Israel die Ehe allerdings zunächst einmal einen ausgesprochen profanen Charakter. Das Alte Testament erzählt von großen Hochzeitsfeiern, von Zärtlichkeit und Erotik (Hohelied) und von der Sorge um die Nachkommenschaft. Bei den Propheten wird dann aber die Ehe auch als Bild für Gottes Treue angesehen. Gott hat mit seinem Volk einen Bund geschlossen, und dieser Bund ist treu und auf Ewigkeit angelegt. Dort, wo sich das Volk Israel gegen Jahwe auflehnt (z.B. beim Durchzug durch die Wüste), wird diese Untreue später bei den Propheten als Ehebruch charakterisiert, z.B. bei Hosea, der im Auftrag Gottes eine Dirne (Hos 1, 2-9) und eine Ehebrecherin (Hos 3, 1-5) zur Frau nimmt, um Israel diese Untreue gegenüber seinem Gott vor Augen zu führen, aber auch um gleichzeitig von der geradezu paradoxen Liebe Gottes zu künden. Denn es gilt immer (was später im NT aufgenommen wird): Selbst wenn der Mensch untreu ist, Gott bleibt doch treu, denn er kann sich selbst und seinen Willen nicht verleugnen.

Jesus hat den Aufruf zu ehelicher Treue radikalisiert, insofern er sie in der Bergpredigt nicht nur der Frau, sondern auch dem Mann als Gebot gibt. Nach jüdischem Gesetz war es dem Mann erlaubt, seine Frau zu entlassen, wenn ein gewichtiger Grund vorlag (über die Gründe, welche die Entlassung legitimieren, stritten sich die Schulen). Jesus verwirft diese Praxis als eine Konzession an die Hartherzigkeit der Männer und fordert die unbedingte Treue der Eheleute, welche es grundsätzlich verbietet, einen Partner fortzuschicken. „Was Gott verbunden hat, das darf der Mensch nicht trennen" $(\mathrm{Mk} 10,9)^{10}$. Jesus revolutioniert in diesem Sinn die Ehepraxis und stellt sich eindeutig auf die Seite der Frau. Es geht ihm dabei allerdings nicht um die Emanzipation der Frau, sondern darum, dass das Gebot Gottes für alle, Frau wie Mann, gilt. Allem Anschein nach sieht er im Bund der Ehe mehr als nur eine weltliche Angele-

9 Vgl. zu diesem Abschnitt v.a. Franz-Josef Nocke, Ehe, in: Handbuch der Dogmatik, Band 2, Düsseldorf 1992, 362-376.

${ }^{10}$ Allerdings gibt es die so genannte „Unzuchtsklausel“ (Mt 5, 32; 19, 9), die im Fall der Unzucht eine Entlassung der Frau legitimiert. Manche Exegeten streiten sich aber auch um die Interpretation dieser Klausel. Als Verwässerung der biblischen Botschaft wird man wohl die Interpretation des EKD-Papiers verstehen müssen: „Das Scheidungsverbot Jesu erinnert die Paare und Eltern an ihre Verantwortlichkeit und macht Kirche und Gesellschaft deutlich, dass Verlässlichkeit für jede Gemeinschaft konstitutiv sind, weil sie die Schwächeren schützen und damit erst den Spielraum für Freiheit und Entwicklung eröffnen“" (Nr. 46). 
genheit, die die Menschen untereinander handhaben können, wie sie wollen. Vielmehr ist die Ehe für ihn Abbild des Bundes Gottes mit den Menschen ${ }^{11}$.

Ein weiteres Abbild-Urbild-Verhältnis ergibt sich aus dem Epheserbrief (Eph 5, 21-32), indem die Liebe zwischen Mann und Frau als Abbild der Liebe Christi zu seiner Kirche verstanden wird. Das „große Geheimnis“ der Liebe zwischen Mann und Frau bezieht Paulus auf Christus und die Kirche und macht damit deutlich, dass neben dem alttestamentlichen Bund Gottes mit dem Volk Israel auch der neutestamentliche Bund Christi mit der Kirche (der ein und derselbe Bund ist) als die unsichtbare Wirklichkeit angesehen werden kann, die in der Ehe sichtbar dargestellt werden soll. Papst Franziskus hat erst kürzlich in einer seiner morgendlichen Predigten in Santa Marta darauf hingewiesen, dass Christus seine Kirche so geliebt hat, dass er sie heiratete. Die eheliche Liebe ist damit ein Abbild der Liebe Christi zu seiner Kirche. „Und vor diesem Weg der Liebe, vor diesem Bild fällt die Kasuistik und wird Schmerz. Wenn aber ... diese Liebe scheitert - denn oftmals scheitert sie - müssen wir den Schmerz des Scheiterns spüren, wie müssen jene Menschen begleiten, die dieses Scheitern in ihrer Liebe erlitten haben. Nicht verurteilen! Mit ihnen gehen! Und ihre Situation nicht zum Gegenstand einer Kasuistik machen"12. So haben wir den Kern des Sakramentes also in der Liebe Gottes zu den Menschen, die sich in Christi Liebe zu seiner Kirche noch einmal manifestiert hat und nun in der Liebe zwischen Mann und Frau zeichenhaft dargestellt werden soll.

\section{Geschichtliche Entwicklung}

Es ist seltsam, dass trotz dieser positiven Konnotation der Ehe im NT die frühe Kirche sich vor allem mit ehefeindlichen Strömungen auseinanderzusetzen hatte. Angestoßen von Paulus und den apostolischen Vätern über die Einsiedler und Wüstenväter galt nämlich sehr bald das jungfräuliche Leben mehr als das Leben in der Ehe. Auch der große Kirchenvater Augustinus, der in dieser Hinsicht für die nachfolgende Zeit maßgeblich geworden ist, hat letztlich ein negatives Verhältnis zur Ehe, oder besser zur Geschlechtlichkeit. Hier zeigt sich der Einfluss von leibfeindlichen Strömungen wie der Stoa und dem Manichäismus. Allerdings lehrt ihn die Bibel auch, dass die Verbindung von Mann und Frau etwas Gutes ist. Gut wird die Ehe nach Augustinus vor allem

${ }^{11}$ Vgl. auch Kasper, Das Evangelium von der Familie, 6: „Es ist Jesu Frohe Botschaft, dass der Bund, den die Eheleute schließen, umschlossen und getragen ist vom Bund Gottes.“

${ }^{12} \mathrm{Vgl}$. die Predigt in http://www.kath.net/news/45086 (Abruf 07.03.2014). 
durch drei „Güter“: die Treue, die Nachkommenschaft und das Sakrament. Sowie der Bund Gottes mit den Menschen durch Treue und Fruchtbarkeit gekennzeichnet war, soll auch die Verbindung von Mann und Frau durch Treue und Nachkommenschaft gekennzeichnet sein. Augustinus entwickelt so die Lehre vom unlöslichen Eheband, das auch dann bestehen bleibt, wenn die Ehe menschlich gesehen zerbricht ${ }^{13}$.

Im Mittelalter begann die Kirche die Ehe stärker zu reglementieren, auch auf Grund der vielen heimlichen Ehen, die geschlossen wurden. So kam es am Ende einer längeren Entwicklung auf dem Konzil von Trient (1545-1563) zur verpflichtenden Eheschließungsform, die mehr oder weniger bis heute gilt. Die Reformatoren kritisierten die Ehejurisdiktion der Kirche und nahmen sie aus dem ganzen sakramentalen Bereich heraus. Auch wenn die Ehe nach Luther der ,edelste Stand“ der ganzen Christenheit ist, nennt er sie letztlich doch ein „weltlich Ding ${ }^{614}$.

Im 20. Jahrhundert wird in der katholischen Theologie ein personal ausgerichtetes Verständnis der Ehe vorherrschend. So wird z.B. neben dem traditionellen Ehezweck (die Erzeugung und Erziehung von Kindern) auch das „beiderseitige Wohl der Ehegatten“ als Ziel der Ehe bezeichnet, d.h. die menschliche Erfüllung der Partner in dieser Lebensgemeinschaft. Man heiratet in diesem Sinn nicht nur, um Nachkommen in die Welt zu setzen, sondern auch, um sich selbst im Partner zu verwirklichen. Es geht also ganz salopp gesagt

${ }^{13}$ Kasper, Das Evangelium von der Familie, 6 kommentiert dazu: „Man darf diese Lehre nicht als eine Art metaphysische Hypostase neben oder über der personalen Liebe der Eheleute verstehen.“

${ }^{14}$ Das EKD-Papier von 2013 begründet aus dem lutherischen Verständnis der Ehe als „weltlich Ding“ die Offenheit und Interpretationsweite derselben in der heutigen Diskussion: „Bei aller Hochschätzung als ,göttlich Werk und Gebot` erklärte Martin Luther die Ehe zum ,weltlich Ding', das von den Partnern gestaltbar ist und gestaltet werden muss. ... Die Ehe ist also für die evangelische Kirche kein Sakrament wie Taufe und Abendmahl; sie ist nicht von Jesus selbst eingesetzt und ist keine absolut gesetzte Ordnung, auch wenn wir uns ihre lebenslange Dauer wünschen. In einem Traugottesdienst feiern wir mit dem Paar, mit Freunden und Familien, dass die beiden , sich getraut', sich den gemeinsamen Weg zugetraut und ihr Leben anvertraut haben, und bitten um Gottes Segen für diese Entscheidung und die gemeinsame Zukunft - nicht mehr, aber auch nicht weniger. Aus diesem evangelischen Verständnis erwächst eine große Freiheit im Umgang mit gesellschaftlichen Veränderungen, die angesichts der Herausforderungen der eigenen Zeit immer wieder neu bedacht und oft auch erst errungen werden muss" (Nr. 48). Hervorhebung CR. 
auch um das Glück von Mann und Frau. So schreibt etwas das Zweite Vatikanische Konzil: „Die christlichen Gatten ... bezeichnen das Geheimnis der Einheit und der fruchtbaren Liebe zwischen Christus und der Kirche und bekommen daran Anteil (vgl. Eph 5, 32). Sie fördern sich Kraft des Sakramentes der Ehe gegenseitig zur Heiligung durch das eheliche Leben sowie in der Annahme und Erziehung der Kinder und haben so in ihrem Lebensstand und in ihrer Ordnung ihre eigene Gabe im Gottesvolk." Allerdings wird deutlich die Hinordnung auf die Familie hervorgehoben: „Aus diesem Ehebund nämlich geht die Familie hervor, in der die neuen Bürger der menschlichen Gesellschaft geboren werden, die durch die Gnade des Heiligen Geistes in der Taufe zu Söhnen Gottes gemacht werden, um dem Volke Gottes im Fluss der Zeiten Dauer zu verleihen. In solch einer Art Hauskirche sollen die Eltern durch Wort und Beispiel für ihre Kinder die ersten Glaubensboten sein und die einem jedem eigene Berufung fördern, die geistliche aber mit besonderer Sorgfalt ${ }^{" 15}$. Trotz der Hervorhebung des Personalaspektes wird also deutlich, dass die Ehe als Keimzelle der Familie von unschätzbarer Bedeutung ist.

\section{Systematische und geistliche Reflexion zur Ehe}

Sakramententheologisch kann man sagen, dass die christliche Ehe realisierendes Zeichen liebender Annahme zu umfassender Lebensgemeinschaft ist, d.h. in ihr verwirklicht sich die liebende Annahme Gottes gegenüber seinem Volk und Jesu Christi gegenüber seiner Kirche. Die liebende Annahme schließt die Gemeinschaft und das Bemühen um das Wohl dessen, den man liebt, ein. Wichtig und spezifisch ist dabei der ganzheitliche Charakter der Ehe:

- Ehe ist nicht nur Ort gemeinsamer Bemühung und geistlicher Kommunikation, sondern auch Ort einer den ganzen Menschen erfassenden, leibhaftigen Begegnung.

- Ehe meint nicht nur begrenzte Interessengemeinschaft, sondern Schicksalsgemeinschaft ,in guten und in bösen Tagen“.

- Ehe meint nicht ein Bündnis für eine begrenzte Zeit („Lebensabschnittsgefährte"), sondern einen Entwurf für das ganze Leben, es geht um eine liebende Annahme zu umfassender Lebensgemeinschaft.

Vor diesem Hintergrund ergibt sich eine Reflexion darüber, was Liebe in der Ehe eigentlich meint. Um mit klassischen griechischen Begriffen zu operieren,

${ }^{15}$ LG 11. Kardinal Kaspar hat übrigens im Nachklang zu seinem Referat vor den Kardinälen gesagt, dass der eigentliche Kern seiner Ausführungen in der Idee der Familie als Hauskirche lag und nicht in der Frage nach den Wiederverheiratet Geschiedenen. 
müsste man sagen, dass die Liebe in der Ehe mehr als nur Eros (die vom anderen ausgehende Faszination und das Verlangen nach dem Einswerden) sein muss - wie wohl diese Dimension für eine gute Ehe von großer Bedeutung ist -, sondern noch mehr Agape (die auf freiem Willensentschluss beruhende Zustimmung zum anderen und zu dessen Wohl). Diese Agape schließt Treue ein: Sie lebt auch dann noch, wenn der "launische“ Eros die Liebenden verlassen hat. So meint ein Leben in ehelicher Liebe nicht einen paradiesischen Zustand von Harmonie und Konfliktlosigkeit, sondern die gemeinsame Suche nach Konfliktlösungen, Vergebung, neues Anfangen, Akzeptanz des Andersseins und auch das Aushalten bleibender Verschiedenheit. Die Liebe in der Ehe bedeutet daher nicht - wie jemand mal richtig gesagt hat -, dass man sich ständig gegenseitig anschaut, sondern dass man gemeinsam nach vorne schaut.

Insofern die Ehe nun aber Sakrament ist, ist sie in der liebenden Annahme auch realisierendes Zeichen der Liebe Gottes. Mit anderen Worten: Es geht in der christlichen Ehe nicht nur um zwei Menschen, sondern auch um Gott. Die Ehegemeinschaft soll in diesem Sinne ,zu dritt“ geführt werden. Eine christlich verstandene Ehe versucht in allen Dingen des Alltags, gerade auch in den Krisen und Schwierigkeiten, Gott mit hineinzunehmen und sich im gemeinsamen Glauben an Gottes Liebe zu orientieren. Dies mag vielleicht ein wenig idealistisch klingen, ist aber für eine christlich gelebte Ehe unabdingbar. Im Unterschied zu einer nicht religiös verstandenen Partnerschaft versteht sich der Bund der Ehe im christlichen Sinn als einen gesegneten Bund, sodass man auf diesen Segen Gottes auch in schwierigen Zeiten vertrauen darf. Konkret könnte das z.B. heißen, Konflikte nicht allein durch Austausch von Argumenten auszutragen, sondern auch durch das Hintragen vor Gott im gemeinsamen Gebet. Durch das konkrete Handeln wird so die Sakramentalität der Ehe deutlich.

Vor diesem Hintergrund stellt sich natürlich die Frage, inwieweit heutige Eheleute sich überhaupt bewusst sind, dass die Ehe ein Sakrament ist und inwieweit sie fähig sind, sie als solche zu leben. Formalrechtlich gesehen ist eine sakramentale Ehe dann geschlossen, wenn beide Partner getauft sind (seien sie katholisch oder evangelisch), allein stellt sich die Frage, ob sie diese Ehe dann auch als Sakrament, d.h. in Offenheit für Gott, leben. Wer lebt seine Liebe zum Partner so, als ob sie ein Abbild der Liebe Gottes zu den Menschen wäre? Wo spielen neben dem Gebet auch die Vergebungsbereitschaft, Annahme von Schuld, soziales Engagement u.a. noch eine Rolle? Sicherlich darf man die Anwesenheit Gottes bzw. die Darstellung seiner Liebe im Ehebund nicht über- 
höhen ${ }^{16}$. Doch darf man sich zumindest des göttlichen Ursprungs der Liebe bewusst bleiben. So sah schon Alfred Delp in seinen tiefen Reflexionen zur Ehe einen Grund für das Sterben der Liebe (woran die Familien zerbrechen) in der Tatsache, dass die Liebe, die viele Menschen füreinander empfinden, ,aus einer unechten Heimat stammt. Wenn zwei Menschen zusammengeraten und ihr Leben auf ein Herz hin wagen und auf ein Schicksal setzen, dann ist das ein Wagnis, dass aus einem Sturm kommen muss, der einmal die Menschen zusammenfügt und zusammenschlägt und zusammenbindet bis ins Allerletzte, wirklich bis zum letzten Herzschlag. Das muss sein, wie wenn zwei Sterne zusammenstoßen, wirklich ein Ereignis, dass bis ins Allerletzte, bis ins Metaphysische, Religiöse hineingeht, bis ins Herz Gottes vorstößt. So ist alle Liebe unecht beheimatet, die aus Berechnung stammt, aus Kalkül, die aufbaut auf Geld, auf Vernunft, auf Konvention, wo nur der Kopf denkt und das Herz schweigt und keine große Flut und kein großer Sturm die Menschen zusammengetrieben haben; oder wenn es nur ein Sturm des Blutes ist, der Triebe, der Wallung, der Sehnsucht, des Hungers, wenn es nicht ein Zusammentreffen wirklich von Mensch zu Mensch ist" ${ }^{\text {"17 }}$.

Zwar kennt Delp auch weitere Gründe für das Scheitern ehelicher Liebe (etwa überzogene Erwartungen an den anderen und die Unfähigkeit, die Liebe im Alltag durchzutragen), den entscheidenden Grund sieht er aber darin, dass die Liebe vieler Eheleute nicht direkt aus dem Herzen Gottes kommt, d.h. man sich zu wenig der göttlich-gnadenhaften Dimension der Ehe bewusst wird. Eine Spiritualität der Ehe kommt nicht ohne Gott aus. Natürlich muss sie auch das Zusammenleben der beiden Menschen, die sich entwickeln und verändern, bedenken. Doch das, was die Ehe positiv als Sakrament ausmacht, ist der göttliche Ursprung. Dieser begegnet auch in den liturgischen Riten bei der Feier der Trauung:

a) Wenn zwei Menschen zueinander ja sagen, geht es zunächst einmal um das Bekenntnis. Ähnlich wie ein Priester sich zu Christus bekennt, sollen sich die Eheleute zueinander bekennen und dies ernst nehmen. In der Liturgie werden einige Fragen gestellt, die mehr Bedeutung haben als den feierlichen Charakter der Eheschließung hervorzuheben. Manchmal hat man den

${ }^{16}$ Vgl. Kasper, Das Evangelium von der Familie, 6: „Als Abbild Gottes ist die menschliche Liebe etwas Großes und Schönes, aber sie ist selbst nicht göttlich. An dieser Überbewertung scheitern viele Ehen."“

${ }^{17}$ A. Delp, Siebenfache Erlösung der Welt, hg. von Roman Bleistein, Frankfurt 1986, 91f. 
Eindruck, dass die Brautleute an dieser Stelle sehr ergriffen sind (weil sie diese Fragen aus der Tradition und aus Spielfilmen kennen), sich aber selten bewusst machen, was damit wirklich verbunden ist. Da sind zum einen die Fragen der Bereitschaft zur christlichen Ehe: zwei werden an jeden Einzelnen gestellt (Frage nach dem freien Entschluss, Frage nach lebenslanger Treue), zwei an das Brautpaar gemeinsam (Bereitschaft zur Annahme von Kindern, Bereitschaft zur Mitverantwortung in Kirche und Welt). Die Liturgie erfährt dann ihren dramatischen Höhepunkt im Vermählungsspruch: „N., vor Gottes Angesicht nehme ich dich an als meine Frau. Ich verspreche dir die Treue in guten und bösen Tagen, in Gesundheit und Krankheit, bis der Tod uns scheidet. Ich will dich lieben, achten und ehren alle Tage meines Lebens" ${ }^{\text {"18 }}$. Machen sich die Brautleute wirklich an dieser Stelle bewusst, was sie da sagen? Zurecht bemerkt Alfred Delp: „Fragen sie sich einmal selbst, was wäre aus mancher Liebe und mancher Familie geworden, wenn diese Fragen wirkliche Antworten gefunden hätten; wenn sich da Menschen nicht selbst belogen hätten oder hätten belügen lassen, sondern in all dem Drang und in all dem Sturm, in all dem großen inneren Drängen diese nüchterne innere Glut gefunden hätten, aus diesem klaren Wissen, aus dieser klaren Sicht sich zu binden an ein endgültiges Ja, bis der Tod scheidet, mit dem ganzen harten Wissen: geschehe, was mag, es wird nicht geschieden, es muss der Tod sein, der scheidet ${ }^{\text {"19 }}$.

b) Bei der Segnung der Ringe wird im Segensgebet die Bitte ausgesprochen, dass in der Gemeinschaft der Liebenden Gottes verborgene Gegenwart unter uns sichtbar werden möge ${ }^{20}$. So wird in den Ringen der Bund Gottes mit den Menschen zeichenhaft-sakramental sichtbar. Zudem wird beim Austausch der Ringe die Wechselseitigkeit der Liebe deutlich. In der alten Trauliturgie wurde dabei gesagt, dass der Bund „zur Ehre Gottes“ und „zum Heil der Seelen“ gestiftet werde. Dadurch war klar, dass es nicht nur um ein paar glückvolle und lustvolle Jahre geht, sondern ein endgültiges Heil ausgesprochen wird.

c) Am Ende des Vermählungsritus legt der Priester die Stola um die Hände der Brautleute und bekräftigt vor den Trauzeugen und den Anwesenden den ehelichen Bund im Namen der Kirche. So wird deutlich, dass die Ehe keine Privatsache ist, ,,weil das Schicksal des Ganzen davon abhängt, was im einzel-

${ }^{18}$ Vgl. Die Feier der Traunn in den katholischen Bistümern des deutschen Sprachgebietes, Freiburg 1992, 62.

${ }^{19}$ Delp, Siebenfache Erlösung, 97.

${ }^{20}$ Vgl. Feier der Trauung, 39. 
nen Raum und Herzen, im einzelnen Haus an Liebe und Gelungenheit oder an Zerbrochenheit und Versagen geschieht ${ }^{\text {" } 21}$.

d) Auch der Trauungssegen ist von entscheidender Bedeutung (besonders wenn es um eine Trauung mit einem orthodoxen Gläubigen geht). In heilsgeschichtlicher Erinnerung an die Treue Gottes zu seinem Volk wird hier die Liebe zwischen Mann und Frau als sichtbarer Ausdruck der Treue Gottes beschrieben.

So ergibt sich aus der Sakramentalität der Ehe insgesamt die spirituelle Sicht auf die Ehe als Ort, an dem Menschen in Gemeinschaft mit Gott zusammenleben und dadurch die Treue und Liebe Gottes in dieser Welt sichtbar werden lassen. Aber ist diese Sicht heute noch vermittelbar? Geht das nicht weit über die Köpfe der Menschen hinweg? Noch wichtiger als eine Spiritualität der Ehe, für die z.B. auch Gedanken von Hans Urs von Balthasar hilfreich sein könnten ${ }^{22}$, ist vielleicht für die Menschen von heute eine Spiritualität in der Ehe für das 21. Jahrhundert. Dazu seien zum Schluss noch einige Punkte gesagt:

- Gebet: Eine gute Ehe lebt vom gemeinsamen Gebet. Es gibt wissenschaftliche Untersuchungen aus den USA, die zeigen, dass Ehen tendenziell seltener geschieden werden, wenn die Eheleute miteinander beten. Das liegt vielleicht nicht nur an der Macht des Gebetes (die nicht in Frage gestellt werden soll), als vielmehr an der Bereitschaft der Betroffenen, schwierige Situationen oder Verletzungen, die man erlitten hat, nicht auszusitzen oder totzuschweigen, sondern vor Gott auszusprechen. Im Gebet etwas auszusprechen ist meist leichter als es jemandem direkt zu sagen. Außerdem zeigt sich im Gebet der Partner der Glaube, dass da jemand ist, der die Beziehung auch in schweren Situationen tragen kann.

- Vergebungsbereitschaft: „Da trat Petrus zu ihm und fragte: Herr, wie oft muss ich meinem Bruder vergeben, wenn er sich gegen mich versündigt? Siebenmal? Jesus sagte zu ihm: Nicht siebenmal, sondern siebenundsiebzigmal“" (Mt 18, 21-22). Ein solches Wort müsste in der Wohnung direkt am Eingang (oder noch besser: in jedem Zimmer) hängen, denn es spielt in der Ehe (aber auch in der Familie allgemein) eine entscheidende Rolle. Viele Ehen scheitern, weil keine Bereitschaft zur Vergebung da ist. In der Ehevorbereitung spreche

${ }^{21}$ Delp, Siebenfache Erlösung, 99.

${ }^{22}$ Vgl. H.U. von Balthasar, Christlicher Stand, Einsiedeln 1977, v.a. 180-202. Balthasar macht mit seiner zugleich schöpfungstheologischen wie christologischen Sicht der Ehe darauf aufmerksam, dass , die Ehe als Ganzes von weiter herkommt und nach weiter hinzielt als eine bloß natürliche Gemeinschaft“ (ebd., 184f). 
ich auch diesen Punkt an. Er ist immer heikel. In der Theorie klingt alles ganz einfach, in der Praxis aber ist es ungemein schwer (vor allem bei Untreue des Partners).

- Freiheit: Es ist gut und wichtig, dass die Eheziele in der neuen Sicht der katholischen Kirche nicht nur auf den Aspekt der Zeugung und Erziehung der Nachkommenschaft Wert legen, sondern auch auf das beiderseitige Wohl von Frau und Mann. Die Ehe ist keine Absage an die viel gepriesene Selbstverwirklichung, sondern eine andere, nicht egoistische Form derselben. Wer in einer Ehe und Familie gewisse Einschränkungen in Kauf nimmt, erfährt zugleich eine große Freude und Freiheit, insofern man nicht mehr auf dem Markt der Möglichkeiten der neuesten Form der Selbstverwirklichung hinterher hecheln muss. Eine Wahl und eine Entscheidung geben auch Sicherheit und Freiheit. Zur Kunst der Freiheit gehört allerdings ebenso, in der Beziehung dem anderen hinsichtlich seiner Freizeitmöglichkeiten oder der Pflege früherer Bekanntschaften Spielraum zu lassen.

- Kinder: Es gibt Ehen, die bleiben ohne Kinder. In vielen Beziehungen sind aber Kinder da und können das Eheleben positiv erfüllen. In den ersten Jahren definiert sich die Beziehung meist über die Kinder (was daran deutlich wird, dass es häufig dann zu Ehekrisen kommt, wenn die Kinder aus dem Haus sind). Kinder sind immer auch ein Geschenk Gottes. In der Bibel (v.a. im AT) wurde dies noch deutlicher gesehen. Kinder als Segen. Wäre diese Sicht nicht auch für heute wiederzugewinnen?

\section{Fazit}

Im Diskurs mit anderen Konzepten partnerschaftlichen Zusammenlebens muss die Kirche zum einen zuhören und unvoreingenommen bestimmte gesellschaftliche Veränderungen wahrnehmen. Zum anderen sollte sie aber auch mutig ihr Konzept von Ehe als lebenslangen Bund mit Gott in die Diskussion einbringen, weil diese weiterhin für die Menschen von heute sinnstiftend und wertevermittelnd ist. In diesem Sinn kann sie auch im eingangs erwähnten Beispiel des Bildungsplans 2015 in Baden-Württemberg ihre Stimme erheben und Gegenvorschläge machen. Johannes Röser hat dies getan und schlägt folgende Bildungsziele für die Schüler vor ${ }^{23}$ :

„Förderung von Ehe und Familie: Schülerinnen und Schüler setzen sich mit der eigenen Sehnsucht nach stabilen, treuen partnerschaftlichen Beziehungen

\footnotetext{
${ }^{23}$ Vgl. CIG Nr. 4 (2014) 43.
} 
und den heutigen Schwierigkeiten, diese zu leben, auseinander, mit dem Ziel, in der Berufsfindung auch Fragen der Familiengründung zu bedenken“.

„Nachhaltige Entwicklung: Schülerinnen und Schüler kennen treue eheliche Beziehungen und reflektieren deren Bedeutung für Staat und Gesellschaft in einer mobilen, globalen Welt".

„Medienbildung: Schülerinnen und Schüler nehmen die Dauer-Präsentation kaputter Ehe- und Familienverhältnisse in Fernsehen, Film und sonstigen Medien als Verletzung der Menschenrechte wahr und erkennen, dass der Einsatz für ein gelingendes Ehe- und Familienleben auch in digitalen Medien ein wesentlicher Bestandteil von Zivilcourage in einer pluralen Gesellschaft ist".

Halten wir fest: Die Ehe ist nach dem Verständnis der katholischen Kirche zwar kein „weltlich Ding“, sie hat aber eine weltliche und gesellschaftliche Funktion. Es gilt, beides miteinander zu denken: den göttlichen Ursprung der ehelichen Liebe, aber auch ihre Bedeutung für die Wertevermittlung in der heutigen Gesellschaft. Eine bleibende Aufgabe ist schließlich der Umgang der Kirche mit dem Scheitern dieser Liebe.

\section{Fr Cornelius Roth: Thing (not-) world. For spiritualities of the marriage during the lack of the sense of direction}

In the today heated discussions are taking place about the marriage at the society, the politics and the Church. Amongst many opinions and the proposal of other forms of life shared the contemporary man often feels lost and doesn't know, which voice he should listen to. With the help for him the Bible is coming. In the Old Testament the marriage i.e. the connection of the man and the woman already appear to the Creator as the gift and has a sacred value, and in the New Testament is a sign of the love of the Christ to the Church. centuries-old theological reflection of the Church is clarifying this mystery.

Key words: spiritualities of the marriage, Church, Bible. 